\section{Chemical Vapor Deposition: It's not just chemistry}

\author{
Chris R. Kleijn \\ Delft University of Technology \\ Department of Multi Scale Physics \\ Prins Bernhardlaan 6 \\ 2628 BW Delft - The Netherlands \\ C.R.Kleijn@TUDelft.NL
}

\begin{abstract}
Although Chemical Vapor Deposition is primarily a chemical process, every CVD experimentalist is (or at least should be) aware of the fact that there is more to CVD than just chemistry. CVD literature is full of observations that cannot be explained from chemical reaction mechanisms and kinetics alone: Deposition rates, uniformities and film properties are found to change when, for instance, (i) one inert carrier gas is replaced by another; (ii) a substrate of different size is used; (iii) substrate rotation is applied; (iv) an identical process is operated in a different reactor setup; etc. All these phenomena have also been observed in low pressure, low temperature CVD processes, which are generally associated with kinetically limited growth.
\end{abstract}

The explanation of the mentioned phenomena is in the gas flow and heat and mass transfer that take place in a CVD reactor. Even under kinetically limited growth conditions, these lead to temperature and concentration gradients within the reactor chamber, which make the relation between set process conditions and actual process conditions near the growth surface ambiguous. This is particularly true for large scale, industrial CVD equipment, but it also plays a role in small scale laboratory reactors. Nevertheless, the influence of reactor geometry and gas flow phenomena is mostly overlooked and rarely reported.

The experimental consequences are large: Kinetic data obtained in one reactor are invalid for another reactor; Processes developed in the laboratory are difficult to scale-up to industrial scale; process conditions reported to lead to good film quality in one reactor fail to do so in another reactor; reactor designs claimed to produce uniform films on $200 \mathrm{~mm}$ wafers are found to be inadequate for $300 \mathrm{~mm}$ wafers, etc.

Also, in extracting kinetic rate constants from experimental data one should be aware of the fact that many kinetic experiments have suffered from gas transport influences.

With the recent interest in Atomic Layer CVD processes, there is a new area of interest for gas flow and transport phenomena in CVD equipment: The minimum cycle time and the degree of uniformity that can be obtained within one cycle directly relate to the extent to which the various ALCVD precursors can be transported to each and every location within the equipment with an operation cycle. The complete removal of precursor A from the reactor chamber before precursor B is introduced is essential in order to prevent the 'pollution' of the pure ALCVD processes by conventional CVD gas phase reactions relates. Dead volumes, gas recirculation zones and the resulting residence time distributions may severely delay full purging.

In this paper we will illustrate the importance of gas flow, heat and mass transfer in CVD equipment. We will also illustrate how mathematical and numerical modeling can be used to separate chemical kinetics from gas flow phenomena, in order to extract true intrinsic kinetics from experimental observations; Furthermore, we will illustrate the importance of gas flow modeling is the scale up of laboratory processes to industrial scale processes, and in the design of equipment fulfilling the high demands in industry with respect to precursor e.g. utilization and film uniformity over large substrates.

For not too low operating pressures, such modeling can be based on the well known continuum equations for gas flow, heat and mass transfer. With the trend to operate CVD at lower and lower pressures, we are however entering a pressure regime in which the molecular nature of the gas should be accounted for. We will discuss methods to do so and their application in modeling very low pressure CVD and ALCVD processes in multi-wafer equipment.

The modeling of gas flow and heat and mass transfer in CVD is essential for the development of industrial size equipment for novel processes. Such modeling, however, is pointless without the inclusion of models for the mechanisms and kinetics of the chemical reactions leading to the transformation of precursor gases into the desired film. The development and publication of such models in a format that can be used in numerical process and equipment modeling is essential, but activities in this field have seriously declined in recent years in favor of studies aimed at the synthesis and study of new precursors alone. The absence of mechanistic chemistry models and kinetic rate constants may seriously hamper the successful industrial implementation of novel CVD processes and precursors. 